\section{AE E T}

ASOCIACIÓN ESPAÑOLA

DE ECOLOGÍA TERRESTRE
Ecosistemas 21(3):100-102 [Septiembre-Diciembre 2012] Doi.: 10.7818/ECOS.2012.21-3.14

Artículo publicado en Open Access bajo los términos de Creative Commons attribution Non Comercial License.

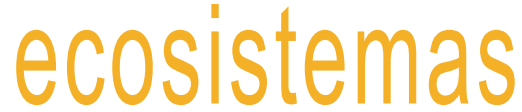

REVISTA CIENTÍFICA DE ECOLOGÍA Y MEDIO AMBIENTE

ISSN 1697-2473 / Open access

disponible en www.revistaecosistemas.net

\title{
La política editorial de Ecosistemas
}

\author{
L. Cayuela ${ }^{1, *}$, Á. Alonso ${ }^{3}$, J.A. Blanco4, C. Puerta-Piñero ${ }^{5}$, S. Rodríguez-Echeverría ${ }^{6}$, L. Jiménez-Eguizabal²
}

(1) Departamento de Biología y Geología, ESCET, Universidad Rey Juan Carlos, c/ Tulipán s/n, 28933 Móstoles, Madrid, España

(2) Revista Ecosistemas. Asociación Española de Ecología Terrestre (AEET), Departamento de Biología y Geología, Universidad Rey Juan Carlos, c/ Tulipán s/n, 28933 Móstoles, Madrid, España.

(3) Departamento de Ciencias de la Vida, Unidad Docente de Ecología, Universidad de Alcalá, Crta. N-II Km 33,6 Campus Universitario, Edificio Biología, 28871 Alcalá de Henares, Madrid, España.

(4) Departamento de Ciencias del Medio Natural, ETSIA, Universidad Pública de Navarra, Campus de Arrosadía s/n, 31006, Pamplona, Navarra, España.

(5) CREAF, Centre de Recerca Ecològica I Aplicacions Forestals, Universidad Autónoma de Barcelona, Bellaterra, E-08193, España.

(6) Centro de Ecología Funcional. Departamento de Ciências da Vida. Universidad de Coimbra. 3000-455. Coimbra. Portugal.

* Autor de correpondencia: L. Cayuela [luis.cayuela@urjc.es]

Cayuela, L., Alonso, Á., Blanco, J.A., Puerta-Piñero, C., Rodríguez-Echeverría., Jiménez-Eguizábal, L. (2012). La Política editorial de Ecosistemas. Ecosistemas 21(3):100-102. Doi.: 10.7818/ECOS.2012.21-3.14

La revista Ecosistemas se ha consolidado en los últimos años como un medio de comunicación científica en lengua castellana en el campo de la ecología y el medio ambiente, con presencia en más de 70 países y un público mayoritario de países latinoamericanos (Navarro et al. 2010) y, recientemente, de Estados Unidos, en donde la comunidad hispanohablante es cada vez mayor. Aunque a lo largo de los últimos años ha llegado a convertirse en un medio de comunicación de calidad, la revista adolece, entre otras cosas, de un perfil un tanto ambiguo (al publicar informes técnicos, trabajos científicos y de divulgación sin agrupar secciones claras) y de una política editorial poco definida. En esta nueva etapa pretendemos que Ecosistemas se consolide como una revista de investigación en lengua castellana, dejando atrás el perfil divulgativo que había tenido inicialmente. El objetivo de esta nota editorial es explicar las razones que nos mueven a redefinir el perfil de la revista, pasando de ser una revista divulgativa científico-técnica a una revista estrictamente científica. Queremos además concretar la política editorial de Ecosistemas, que será la base sobre la cual el comité editorial decidirá qué contribuciones serán publicadas en la misma.

\section{La evolución de Ecosistemas}

Aunque aparentemente convertir una publicación divulgativa en una publicación científica supone un cambio importante para una revista como Ecosistemas, queremos resaltar que dicho cambio es más de forma que de fondo. Ello es porque Ecosistemas, desde nuestro punto de vista, ya era una revista eminentemente científica, en la que la parte puramente divulgativa ocupaba un espacio cada vez menor. Además, la revista ha ido evolucionando de manera natural en los últimos años, pasando de tener un perfil muy amplio a ser una publicación científica de referencia en lengua castellana (Fig. 1). Tal vez sea más fácil entender esto si conocemos la historia de la revista.

La revista surge inicialmente en 1991 como un boletín informativo de la Asociación Española de Ecología Terrestre (AEET) ( Navarro et al. 2010). A finales de ese mismo año, se decide ampliar el contenido del mismo y adoptar el formato de revista de divulgación. Así pues, Ecosistemas nace como una revista de divulgación científica y técnica de ecología y medio ambiente y con una periodicidad trimestral. En el último trimestre de 1992 se publica el primer ejemplar impreso de Eco- sistemas con el número 3 e ISSN 1132-6344, se sigue este sistema de numeración hasta finales de 1998 ( $\left.n^{\circ} 26\right)$. A partir del año 1999 se cambia el sistema de numeración y se inicia el contaje por el sistema número de trimestre/año natural.

A pesar de convertirse en referente de la divulgación científica en España durante estos años, los gastos que generaban su producción y distribución no eran cubiertos por los ingresos, por lo que su edición impresa tuvo que ser cancelada en el año 2000. El último número editado en soporte papel es el ( $\left.n^{0} 1 / 2000\right)$, correspondiente al periodo abril-junio (Fig. 2). A partir de enero de 2001 se relanza la revista, se cambia el tipo de soporte, se edita en formato electrónico con ISSN 1697-2473, se cambia la periodicidad a cuatrimestral, se recomienza el contaje con la fórmula volumen $=$ año publicación $\left(n^{\circ}\right.$ cuatrimestre) siendo el primer número de esta etapa el 10(1) y se constituye un nuevo comité editorial formado por investigadores. Aunque existe una manifiesta intención por parte del nuevo comité editorial de mantener un perfil divulgativo, el salto al formato electrónico supone ya de partida un cambio sustancial que se refleja en un claro predominio de trabajos de índole estrictamente científica y no divulgativa. Como ejemplo significativo citaremos que ya en estos comienzos se impone el sistema de revisión por pares para los artículos de investigación y de revisión, algo que es característico de las publicaciones científicas, y que está ausente de los artículos de índole más divulgativa, cuyo rigor suele estar garantizado básicamente por la experiencia en el tema del autor. Si nos fijamos en los contenidos de muchas de estas primeras contribuciones electrónicas, es fácil notar un cambio sustancial con respecto a las contribuciones publicadas en la versión en papel: abundan los tecnicismos y cultismos, la audiencia a la que se dirigen muchos de estos trabajos es claramente especializada (o al menos académica), y la estructura del texto es propia de trabajos científicos.

Este carácter marcadamente científico con el que nace la versión electrónica de Ecosistemas se va acentuando con el tiempo (ver Navarro 2009). En la Figura 1 se puede ver la evolución de las distintas secciones de la revista, separadas por grandes bloques en contribuciones de carácter científico (artículos de investigación, artículos de revisión, resúmenes de tesis doctorales), de carácter técnico (informes, revisiones sobre la ecología de una especie, resúmenes de proyectos o actividades sobre educación ambiental) y de carácter divulgativo (apuntes, notas y foro, artículos de opinión, entrevistas). 
Contribuciones de carácter científico

Articulos de investigación Alltul.III

$2001 \quad 2004 \quad 2007 \quad 2010$

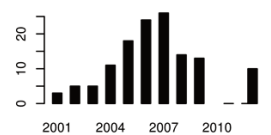

Resúmenes de tesis

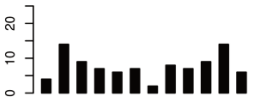

\section{0}

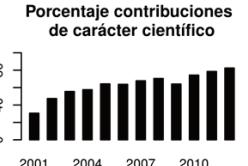

Contribuciones de
carácter técnico

Informes

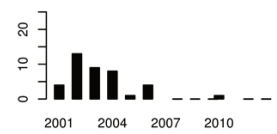

Especies

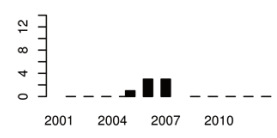

Educación ambiental
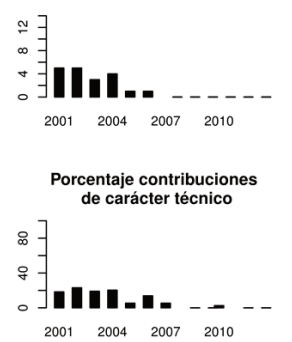

Contribuciones de carácter divulgativo

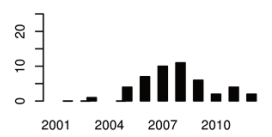

$\begin{array}{lll}2001 & 2004 \quad 2007 \quad 2010\end{array}$

Opinión

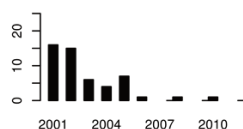

Entrevistas

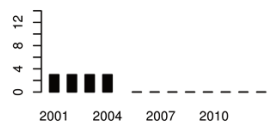

Porcentaje contribuciones de carácter divulgativo

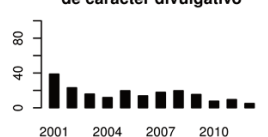

Apuntes, Notas y Foro

Figura 1. Evolución temporal del número de trabajos publicados en Ecosistemas por secciones. Las secciones han sido agrupadas por grandes bloques en: contribuciones de carácter científico (artículos de investigación, artículos de revisión, resúmenes de tesis doctorales; izquierda), de carácter técnico (informes, revisiones sobre la ecología de una especie, resúmenes de proyectos o actividades sobre educación ambiental; centro) y de carácter divulgativo (apuntes, notas y foro, artículos de opinión, entrevistas; derecha). Las gráficas de la parte inferior muestran los porcentajes de cada tipo de contribución (científicas, técnicas, divulgativas) con respecto al total de trabajos publicados cada año en la revista.

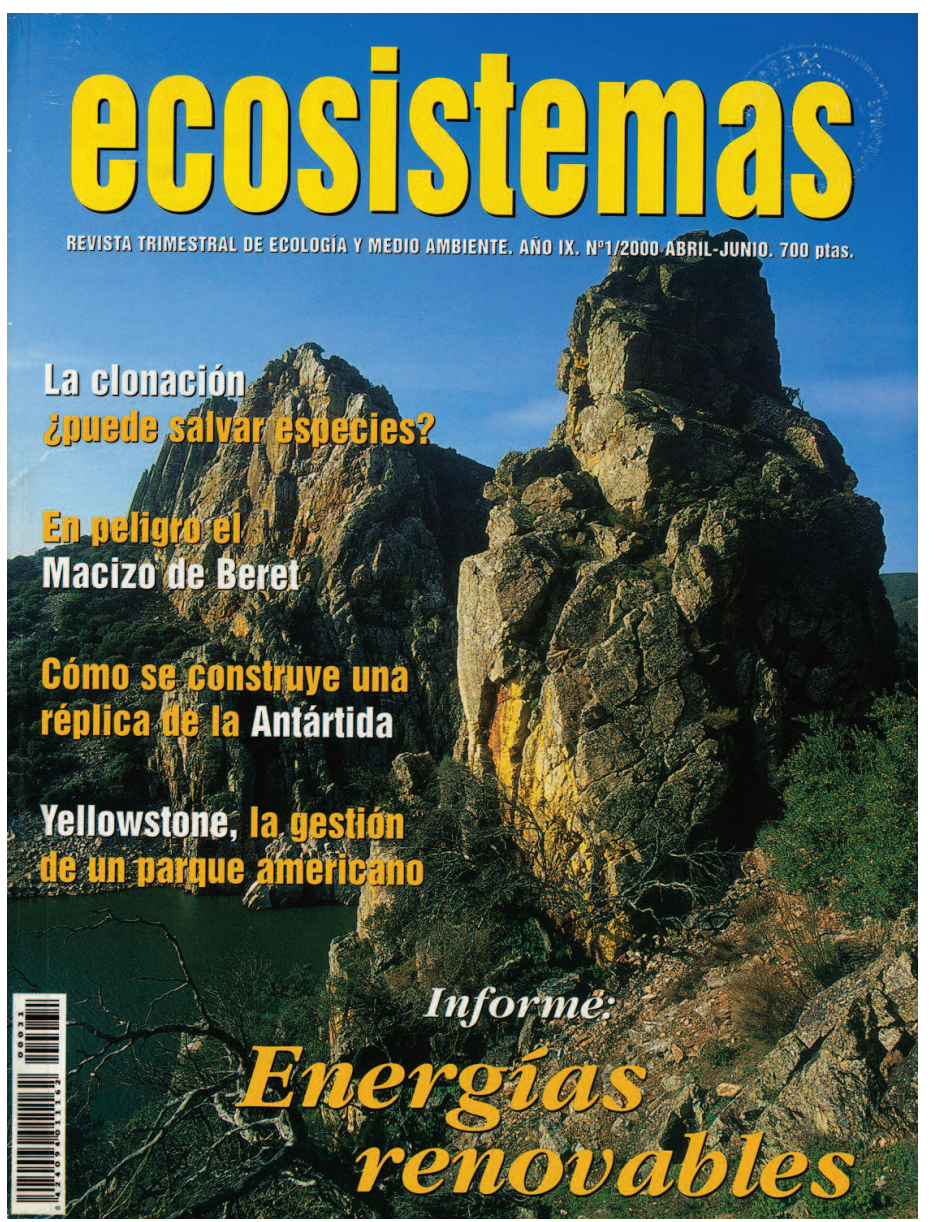

Figura 2. Portada del úlltimo número publicado en papel de la revista Ecosistemas, en Abril-Junio de 2000.
Como se puede ver en esta figura, el porcentaje de contribuciones de carácter científico va aumentando de forma progresiva desde el $30 \%$ en el año 2001 hasta algo más del $80 \%$ en el año 2012. Al contrario de lo que ocurre con las contribuciones de carácter científico, las contribuciones de carácter técnico y divulgativo que se han publicado en la revista, han ido disminuyendo de forma progresiva a lo largo de estos años. Algunos tipos de contribuciones, como las entrevistas, los artículos de opinión o los artículos sobre la ecología de especies o resúmenes de proyectos o actividades relacionados con la educación ambiental, desaparecen por completo de la línea editorial de la revista en los últimos años, sin que haya un reflejo de estos cambios en la política editorial. Esta evolución posiblemente refleja el cambio en los intereses de los propios lectores, que a menudo también son los autores de los artículos publicados. Aunque las secciones tienen mucha variabilidad en cuanto a la naturaleza de sus contenidos, creemos que este análisis somero puede servir como una aproximación que ilustra bastante bien la evolución de la revista en la última década. Con base en sus contenidos podemos decir que Ecosistemas se ha convertido, por tanto, en los últimos años una revista eminentemente científica.

\section{Nuevos criterios para publicar en Ecosistemas}

Consolidando la línea de los anteriores comités editoriales y que ha marcado una de las especiales características de la revista, los futuros números de Ecosistemas estarán, cada uno de ellos, articulados alrededor de un monográfico de artículos con un tema común. Los artículos pertenecientes al monográfico estarán coordinados por uno o varios Editores Invitados, expertos en el tema a tratar. Éstos invitarán a autores a enviar contribuciones al monográfico, pero cada uno de los monográficos también estará abierto a otros autores que quieran participar en los mismos y que no hayan sido contactados por los Editores Invitados. Nuestro objetivo es que los artículos pertenecientes al monográfico sean aproximadamente la mitad de las aportaciones de cada número, siendo el resto trabajos de tema libre enviados directamente por los autores al comité editorial permanente de la revista.

Para afianzar su perfil como revista de investigación, el nuevo equipo editorial ha definido una serie de criterios para concretar el tipo de trabajos que podrán ser publicados en Ecosistemas. Específicamente se propone que:

- Todos los artículos publicados, ya sean artículos de investigación, revisiones o comunicaciones breves, deben ser inéditos.

- Se aceptarán artículos únicamente en idioma castellano, aunque todos los artículos tendrán el resumen en castellano e inglés.

- Los trabajos descriptivos sin una interpretación y discusión detallada del significado ecológico que puede extraerse de los resultados escritos, los que reflejen una opinión personal, o que sean puramente especulativos sin resultados claros que apoyen las conclusiones descritas, serán rechazados a nivel editorial.

- Los artículos de investigación deberán tener unos objetivos claros y una metodología bien definida. Las preguntas e hipótesis tienen que estar planteadas de forma concisa al final de la introducción. La metodología tiene que ser apropiada para resolver las cuestiones planteadas en los objetivos. Este será uno de los puntos principales que los revisores invitados comprobarán a la hora de determinar su opinión sobre el mérito de la contribución enviada a la revista.

- En el caso de artículos de revisión que sinteticen los resultados de trabajos previos del autor o autores, se espera que éstas tengan un valor añadido y aporten ideas o mensajes que no se encuentre en los trabajos anteriores. Siguiendo la tradición de la revista de proporcionar los últimos avances en la ciencia de la ecología y medio ambiente, los artículos de revisión seguirán siendo una parte importante de la revista. Creemos que el escribir este tipo de artículos en castellano facilitará un primer acceso a la literatura científica en el mundo hispanohablante. La mayoría de artículos de revisión se enviarán a la revista por invitación, normalmente como parte de un monográfico sobre un tema específico que centrará cada uno de los números de la revista. Sin embargo, los artículos de revisión envia- 
dos por autores que no hayan sido previamente invitados por los editores también serán bienvenidos.

- Dada la naturaleza puramente digital de la revista no habrá limitaciones en el número de artículos por monográfico, de forma que todos los artículos enviados para formar parte de un monográfico y que su proceso de revisión sea favorable dentro del tiempo estipulado serán incluidos en el número monográfico. Sin embargo, si por algún motivo no fuera posible la inclusión de un artículo aceptado en el monográfico en cuestión, se invitará a los autores a que publiquen su contribución en el siguiente número de Ecosistemas como una contribución regular.

- La nueva sección de comunicaciones breves surge para dar cabida a trabajos incipientes, reflexiones sustentadas sobre evidencias científicas pero todavía no formalizadas en un trabajo de investigación, la presentación de trabajos de índole más técnica (p.ej. desarrollo de una nueva metodología en cualquier disciplina relacionada con la ecología o el medio ambiente), aplicaciones de investigaciones ya realizadas a la gestión y conservación del medio natural, o comentarios a artículos publicados anteriormente en Ecosistemas.

Estos criterios se pueden resumir en una serie de preguntas que los autores deberán hacerse antes de enviar su trabajo a Ecosistemas:

1.- ¿Encaja el tema abordado dentro del contexto de la ecología o el medio ambiente?

2.- ¿Se sustentan los resultados en observaciones o experimentos realizados utilizando las técnicas experimentales y de tratamiento de datos adecuadas?

3.- ¿Supone el trabajo algún avance para su área de conocimiento?

4.- ¿Deja el artículo suficientemente claro qué contribución hace a su área de conocimiento en relación al conocimiento ya establecido o a los resultados de trabajos previos?

Además, los autores que persigan publicar sus trabajos en Ecosistemas deberán hacerse las siguientes preguntas en relación a la estructura de su trabajo:

5.- ¿Refleja el título fielmente el contenido del artículo?

6.- ¿Se ajusta el resumen adecuadamente al contenido del artículo?

7.- ¿La introducción está bien estructurada y sitúa el estudio en contexto?

8.- ¿Están definidos los objetivos de forma clara y concisa?

9.- ¿Son adecuados los métodos utilizados y han sido aplicados correctamente?

10.-¿Son adecuadas las figuras y tablas en número y calidad?

11.-¿Están los resultados explicados adecuadamente y se discuten en un marco amplio?

12.-¿Son las referencias apropiadas en número y relevancia?

13.-¿Es el texto conciso y está escrito correctamente?

Todas estas preguntas serán consideradas en el proceso de revisión. Si las respuestas a las preguntas 1 a 4 es positiva en ningún caso se rechazará el artículo y se podrán requerir cambios menores o sustanciales dependiendo de si algunas de las respuestas a las preguntas 5 a 13 haya sido "no". La calidad del proceso de revisión estará asegurada por la participación desinteresada de los revisores, miembros de la comunidad científica a la que también está dirigida la revista (Lajtha y Baveye 2010). Por lo tanto, queremos aprovechar a hacer un llamamiento a los científicos que puedan recibir en el futuro una invitación a revisar artículos para Ecosistemas para que las acepten, dentro de sus posibilidades de disponibilidad de tiempo y conocimiento de las técnicas utilizadas en el manuscrito a revisar. La participación en el proceso de publicación como revisor asegura la supervivencia de la revista al mantener su calidad (McPeek et al. 2009), y es una forma de solidaridad entre colegas (Navarro et al. 2010). Todos los científicos que quieran publicar artículos deben considerar que al menos habrá dos revisores y un editor asignados a su manuscrito. Dado el relativo pequeño tamaño de la comunidad científica hispanohablante en ecología y medio ambiente, si la mayoría de las invitaciones a revisar artículos fueran rechazadas, el comité editorial se quedaría muy pronto sin opciones que mantengan una calidad de la revisión

\section{No todo es ciencia en la vida: el papel de la divulgación}

Como ya hemos especificado, la razón por la que Ecosistemas se consolida a día de hoy como revista de investigación, no es necesariamente porque pensemos que la investigación es más importante que la divulgación. Más bien al contrario, los científicos cada vez somos más conscientes del importante papel que juega la divulgación a la hora de transferir el conocimiento generado por la ciencia a la sociedad (García-Fayos et al. 2008). Se trata más bien de una cuestión de limitaciones. Los científicos somos buenos haciendo ciencia, pero salvo algunas honrosas excepciones, no somos tan buenos como divulgadores. Es por ello por lo que hacemos lo que mejor sabemos hacer y dejar la divulgación a los expertos en esta materia. A pesar de esto, no queríamos dejar de lado el motivo que causó el nacimiento de la revista, pero dándole un formato distinto, aprovechando el nuevo desarrollo de las tecnologías de comunicación (Wilkins 2008) y la capacidad dinámica de los recursos digitales en los que se publica la revista. Ecosistemas ya no acepta contribuciones que sean divulgativas. Sin embargo, queremos que los contenidos divulgativos sean una parte importante de la experiencia de los lectores que accedan a la revista. Por lo tanto, hemos incorporado al equipo editorial en esta nueva etapa a una editora de comunicación, Nieves Martín, que se encargará de comentar en clave divulgativa algunos de los trabajos más relevantes que publiquemos en Ecosistemas. Ello se hará por medio de un blog asociado a la revista (http://revistaecosistemasblog.net), con lo que esperamos contribuir a un doble objetivo: (1) continuar haciendo divulgación, pero esta vez en un medio específico y bien definido, que funcione en paralelo a la revista; y (2) aumentar la visibilidad de los artículos publicados en la misma.

\section{El éxito de Ecosistemas: una tarea de todos}

Para finalizar, desde el nuevo comité editorial queremos, en primer lugar, reconocer la importante labor de los anteriores comités en garantizar el éxito y la supervivencia de la revista hasta que la responsabilidad de la misma ha caído en nuestras manos. En segundo lugar, queremos recordar que aunque nosotros coordinemos el día a día de la publicación, el éxito de Ecosistemas dependerá principalmente del interés que muestren en la revista los lectores. Serán ellos los que primero sigan utilizando la revista para acceder a resultados de investigación, algo que animará a algunos de los lectores a ser también autores y enviar contribuciones a la revista. $Y$ también entre los lectores estarán los revisores que aseguren la calidad científica del trabajo publicado. En definitiva, esperamos que el nuevo formato, orientación y herramientas asociadas a la revista sean del agrado y del interés de los lectores.

El Comité Editorial de Ecosistemas

Diciembre de 2012

\section{Referencias}

García-Fayos, P., Bonet, F.J., Valladares, F., Traveset, A., Pausas, J.G., Imbert, J. B., Lloret, F. 2008. El ecólogo en su laberinto. Ecosistemas 17(2):125-127.

Lajtha, K., Baveye, P.C. 2010. How should we deal with the growing peer-review problem? Biogeochemistry 101:1-3.

McPeek, M.A., De Angelis, D.L., Shaw, R.G., Moore, A.J., Rausher, M.D., Strong, D.R., Ellison, A.M., Barrett, L., Rieseberg, L., Breed, M.D., Sullivan, J., Osenberg, C.W., Holyoak, M., Elgar, M.A. 2009. The golden rule of reviewing. American Naturalist 173:155-158.

Navarro, L. 2009. La calidad de la información en las revistas de acceso abierto. Ecosistemas 18(2):1-2.

Navarro, L., Sánchez, J.M., Cortina, J., Jiménez-Eguizábal, L. 2010. En manos de la buena voluntad de los colegas... En nuestras manos. Ecosistemas 19(1):1-7

Wilkins, J.S. 2008. The roles, reasons and restrictions of science blogs. Trends in Ecology and Evolution 23(8):411-413. 